\section{Evaluation of Different Levels Thyroid Dysfunction in Patients having Diffuse Goiter from Rawalpindi and Islamabad}

\section{Abstract}

The enlargement of the thyroid gland is a common problem in clinical practice associated with iodine deficiency, increase in serum Thyroid-Stimulating Hormone (TSH) level, natural goitrogens, smoking, and lack of selenium and iron. Diffuse goiter is characterized by the enlargement of whole thyroid gland at the age of puberty. It is observed in the patients either having hypothyroidism or hyperthyroidism or have abnormal TSH concentrations. It has investigated that Diffuse goiter has been exhibiting equivalence with diffuse radioactive iodine uptake. The data comprised of 144 patients of diffuse goiter evaluated through various statistical techniques in order to investigate various thyroid dysfunctioning based on the normal concentration of thyroid function tests. Hypothyroidism and hyperthyroidism can be accurately diagnosed with laboratory tests performed at NORI. The data along with detailed history was collected with the help of clinicians in NORI and entered in the Performa. The diagnostic evaluation relating to functional and morphological characterization has examined through serum TSH concentration and imaging. The result shows non-significant difference between hypothyroid and hyperthyroid patients of diffuse goiter and control group individuals. The study has concluded that the prevalence of diffuse goiter is not dependent on the higher and lower concentration of TSH.

Keywords: Hyperthyroid; Hypothyroid; Thyroid-Stimulating Hormone (TSH); Serum

\author{
Muhammad Ameen* \\ Arid Agricultural University, Rawalpindi, \\ Pakistan
}

\section{*Corresponding author: Muhammad Ameen}

- muhammadameen_1@live.com

Arid Agricultural University, Rawalpindi, Pakistan.

Tel: 03005839161

Citation: Ameen M (2017) Evaluation of Different Levels Thyroid Dysfunction in Patients having Diffuse Goiter from Rawalpindi and Islamabad. J Clin Epigenet. Vol. 3 No. 3:31

\section{Introduction}

Thyroid gland is a highly vascular endocrine gland that is placed anteriorly in the neck, extending from the fifth cervical to the first thoracic vertebrae [1] have investigated that in case of mild hypothyroidism serum TSH also have been observed high and the values of $\mathrm{T} 3$ and $\mathrm{T} 4$ have remained in normal range.

Diffuse goiter is characterized by the enlargement of whole thyroid gland is observed in the patients either having hypothyroidism or hyperthyroidism at the age of puberty while toxic diffuse goiter is also known as grave's disease is prevalent in hyperthyroid patients [2] have reported that Diffuse Goiter (Simple) or physiological goiter is the most common thyroid disorder among different thyroid diseases. They have further investigated that Diffuse goiter has been exhibiting equivalence with diffuse radioactive iodine uptake, while Multinodular goiter has not been exhibiting equivalence with irregularity in uptake and activity throughout the thyroid gland. Schwartz CE [3] have observed the two basic forms of thyroid hormone that are T4 (3,5,3', $5^{\prime}$-tetraiodothyronine) and T3 (3, 3',5-triiodothyronine), which have been produced and secreted by the follicular cells of the thyroid gland. They have further revealed that these hormones have played extremely important role in the normal development of cellular metabolism, cell proliferation and cell differentiation. Surks and Hollowell have described that a thyroid disorder is physical ailment that arises from its dysfunctioning that produces Triiodothyronine (T3) and Thyroxin (T4). They have further observed that this dysfunctioning have influenced functions of the pituitary gland and hypothalamus and consequently their secretions [4]. Oppenheimer et al. [5], have revealed that Tetraiodothyronine (T4) is generated by the thyroid gland as an inactive compound. They have further described that this is transformed into the tissues to triiodothyronine (T3) that combines to the nuclear receptors in order to initiate thyroid hormone (TH) action. Guyton and Hall have analyzed that thyroid 
gland have produced about 7\% Triiodothyronine (T3) and about 93\% thyroxin (T4). Triiodothyronine (fT3) has 3-5 folds greater biological potency than thyroxin (fT4). Morreale de Escobar et al. [6] have reported that thyroid hormones as being critical regulatory molecules that played an important role in functioning and development of various organs of vertebrate body particularly normal brain functioning before and after birth and further it has been examined that these are Tyrosine based hormones that are partially composed of iodine and their deficiency enlarges the thyroid tissue which is known as simple goiter [7]. Suzuki et al. [8] have examined that alteration in TSH and free thyroid hormones have been observed in gender and during aging. They have further investigated that in males the process of aging have restrained the free thyroid hormones concentration but have not influenced on the concentration of TSH. While, in females the free thyroid hormones levels have not been changed with aging but TSH level have been increased in age-dependent areas of severe iodine deficiency can be as high as $80 \%$. Khan et al. [9] have observed that two major ailments of thyroid gland, one is higher generation of thyroid hormones that is called hyperthyroidism and other is lower generation of thyroid hormones that is called hypothyroidism [9] also have examined that serum TSH level has been elevated in case of primary hypothyroidism but values of T3 and T4 have remained below the normal range. Caldwell et al. have been investigated that in case of mild hypothyroidism serum TSH also have been observed high and the values of T3 and T4 have remained in normal range [10] have been reported that in case of hyperthyroidism the values of triiodothyronine and Thyroxin have been observed above high level and TSH has subdued due to negative feedback mechanism. Current paper will explore various medical disorders and abnormalities in thyroid hormones in Pakistani population. Further, profile of thyroid hormones will be analyzed via level of different hormones and antibodies like TSH, FT4, T3 and ant thyroglobulin respectively will be analyzed from serum samples of patients. Analytical evaluation of the hormonal profile of patient will be carried out via electrochemical luminescent immunoassays (ECLIA). The objective of study is to compare the hormonal profile of subclinical and clinical hypothyroid patients with normal healthy individuals and will be evaluated statistically.

\section{Materials and Methods}

The current study was carried out at Nuclear Medicine, Oncology and Radiotherapy Institute (NORI) in patients of diffuse goiter and control group individuals having no diffuse goiter. The population sample is comprised of 194 individuals. The whole population was categorized into two major groups, one is experimental group and other is control group. The blood tests reports showing normal values of Thyroid Functions Tests (TFTs) were designated as normal while patients having not normal values of TFTs were designated as abnormal. The experimental group constituted 144 patients of diffuse goiter and 50 individuals as a control group. In a whole sample of population 79 were observed as normal and 65 as abnormal patients. The abnormal patients were further categorized into hyperthyroid and hypothyroid according to the values of TSH levels. Hypothyroidism and hyperthyroidism can be accurately diagnosed with laboratory tests performed at NORI. The patients having higher concentration of TSH were designated as hypothyroid and patients having low concentration of TSH were designated as hyperthyroid. Individuals having no diffuse goiter were taken as control group but they can have hypothyroidism or hyperthyroidism. The patients having diffuse goiter were diagnosed through Physical examination, clinical examination, Thyroid Scan, and Ultrasound reports. The blood tests reports of thyroid function tests were collected from various laboratories of Pakistan and normal TSH concentration ( 0.27 to $4.2 \mu \mathrm{lU} / \mathrm{ml})$ was kept as criteria for the diagnosis of thyroid dysfunction.

\section{Setting and Study Area}

The present study was carried out in Nuclear Medicine, Oncology and Radiotherapy Institute (NORI). Study population: Total 194 patients that were referred for thyroid scan were included in the study. Inclusion Criteria: All patients having diffused goiter in any age group will be included in the study.

Exclusion criteria:

- Pregnancy.

- Liver disease as evidenced by raised ALT level.

- Drugs: Amiodarone, lithium, anti-thyroid medications (neomercazole/procarbizole), thyroxin.

\section{Study Group}

The whole population sample constituting the patients of diffuse goiter was based on the incidence of Thyroid Functions Tests (TFTs).

\section{Data Collection}

After having informed consent (Annexure-I) from patients, the data along with detailed history was collected with the help of clinicians in NORI and entered in the Performa (Annexure-II).

\section{Data Analysis}

The data was analyzed using SPSS version 17 . The average values for age, history of goiter (duration) etc. was calculated. The percentage of different thyroid dysfunctions (hypothyroid, hyperthyroid) in patients of diffuse goiter was analyzed through confidence interval (0.05). Statistically defined P-value of the study is (0.000). The study has considered Thyroid Stimulating Hormone (TSH) as a criterion for the diagnosis of thyroid dysfunction. The normal range of Thyroid Function (TFTs) regarding TSH diagnosed at NORI hospital is $(0.27-4.2 \mu \mathrm{lU} / \mathrm{ml})$ and other clinical laboratories of Pakistan. The patients having high TSH concentration have been designated as Hypothyroid and the patients having low serum TSH concentration have been designated as Hyperthyroid while the patients having normal TSH values are considered as thyroid.

\section{Results}

The current paper has investigated that $55 \%$ have abnormal TSH concentration, while $45 \%$ have normal TSH concentration in a whole sample of population having diffuse goiter. In abnormal patients, about $71 \%$ have low TSH levels, while $29 \%$ have high TSH levels. TSH levels ranging from 0.27-4.2 $\mu \mathrm{IU} / \mathrm{ml}$ was considered as normal for the diagnosis in thyroid function tests (TFTs). When the abnormal patients of diffuse goiter 
having the clinical symptoms of hypothyroid or hyperthyroid were compared with control group individuals, statistically nonsignificant difference was observed in this case. Comparatively, similar results were obtained in the studies of nodular goiter and multinodular goiter. The study has worked out to determine the comparative evaluation of thyroid function tests between normal and abnormal, hyperthyroid and hypothyroid of diffuse goiter, Nodular Goiter (NG), Multinodular Goiter (MNG) and control group. The statistical evaluation of thyroid function tests in the group having diffused goiter has done in the statistical evaluation table (Tables 1 and 2 ).

The following above tables have shown the statistical difference between abnormal, hypothyroid and hyperthyroid patients versus control individuals. The abnormal TSH levels patients (55\%) and normal TSH levels patients (45\%) of diffuse goiter have shown that incidence of diffuse goiter is not only dependent on thyroid dysfunction tests. Moreover, among abnormal patients $71 \%$ low TSH levels and 29\% high TSH levels have revealed the fact diffuse goiter is dependent on the concentration of TSH. The patients with low TSH levels have greater chance of occurrence of disease as compared to patients with high TSH levels. Comparison of lipid profile in euthyroid, subclinical, and clinical hypothyroid and hyperthyroid versus control group individuals showed non-significant differences by non-parametric tests $(p>0.05)$. Similar results were obtained pertaining to nodular goiter and multinodular goiter. Hypothyroid, euthyroids, Clinical and subclinical hypothyroid and hyperthyroid patients of diffuse goiter and MNG have not altered lipid profile of control individuals contrary to euthyroids of nodular goiter. However, thyroid

Table $1 \mathrm{X}^{2}$ calculations (Two-way Contingency) showing statistical difference between hypothyroid, hyperthyroid and normal TSH patients of diffuse goiter versus control individuals.

\begin{tabular}{|c|c|c|c|c|c|}
\hline $\begin{array}{l}\text { Number of } \\
\text { Patients with TSH } \\
\text { Concentrations }\end{array}$ & Total No. & Male & Female & Control & P-value \\
\hline Normal & $\begin{array}{c}79 \\
70.34 \\
(1.07)\end{array}$ & $\begin{array}{c}13 \\
16.16 \\
(0.62)\end{array}$ & $\begin{array}{c}66 \\
54.19 \\
(2.57)\end{array}$ & $\begin{array}{c}50 \\
67.31 \\
(4.45)\end{array}$ & 0.1163 \\
\hline Abnormal & $\begin{array}{c}65 \\
60.87 \\
(0.28)\end{array}$ & $\begin{array}{c}17 \\
13.98 \\
(0.65)\end{array}$ & $\begin{array}{c}48 \\
46.89 \\
(0.03)\end{array}$ & $\begin{array}{c}50 \\
58.25 \\
(1.17)\end{array}$ & 0.8254 \\
\hline High & $\begin{array}{c}19 \\
29.76 \\
(3.89)\end{array}$ & $\begin{array}{c}5 \\
6.83 \\
(0.49)\end{array}$ & $\begin{array}{c}14 \\
22.93 \\
(3.47)\end{array}$ & $\begin{array}{c}50 \\
28.48 \\
(16.26)\end{array}$ & 0.8919 \\
\hline Low & $\begin{array}{c}46 \\
48.02 \\
(0.09)\end{array}$ & $\begin{array}{c}13 \\
11.03 \\
(0.35)\end{array}$ & $\begin{array}{c}33 \\
36.99 \\
(0.43)\end{array}$ & $\begin{array}{c}50 \\
45.95 \\
(0.36)\end{array}$ & 1.1157 \\
\hline
\end{tabular}

$\chi^{2}=36.183, d f=9, \chi^{2} / d f=4.02, P\left(\chi^{2}>36.183\right)=0.0000$

\section{References}

1 Dixit D, Shilpa MB, Harsh MP, Ravishankar MV (2009) Agenesis of isthmus of thyroid gland in adult human cadavers: a case series. Cases J 2: 6640

2 Kumar V, Cotran RS, Robbins SL (1997) Basic pathdogy (6 ${ }^{\text {th }}$ edn.). W.B. Saunders and co pp. 643-652.
Table $2 \mathrm{X}^{2}$ calculations (Two-way contingency) showing statistical difference between hypothyroid, hyperthyroid and normal TSH patients of Nodular Goiter (NG) versus control individuals.

\begin{tabular}{|c|c|c|c|c|c|}
$\begin{array}{c}\text { Number of } \\
\begin{array}{c}\text { Patients with TSH } \\
\text { Concentrations }\end{array}\end{array}$ & $\begin{array}{c}\text { Total } \\
\text { No. }\end{array}$ & Male & Female & Control & P-value \\
\hline Normal & 131 & 12 & 119 & 50 & \\
& & $18.82(2.47)$ & 112.18 & 50.00 & 0.0012 \\
\hline & & 11 & 42 & $(0.00)$ & \\
\hline Abnormal & 53 & 12.86 & 40.14 & 50.00 & 0.3913 \\
& & $(0.27)$ & $(0.09)$ & $(0.00)$ & \\
\hline & & 9 & 37 & 50 & \\
\hline High & 46 & 11.02 & 34.98 & 50.00 & 0.3334 \\
& & $(0.37)$ & $(0.12)$ & $(0.00)$ & \\
\hline & & 2 & 5 & 50 & \\
\hline Low & 7 & 1.96 & 5.04 & 50.00 & 1.0926 \\
& & $(0.00)$ & $(0.00)$ & $(0.00)$ & \\
\hline
\end{tabular}

dysfunctioning was not prominent in patients experiencing diffuse goiter and MNG. The result revealed that TSH, FT3 and FT4 levels did not affect the onset goiter in both groups. Similarly, the study is correlated with the findings of Kumar et al. investigated that Diffuse goiter has been exhibiting equivalence with diffuse radioactive iodine uptake. The prevalence of disease is not dependent upon the concentration of TSH. Therefore, the research work has inconsistency with the findings of Dr. Firdushi Begum that estimation of serum thyroid hormones and TSH concentrations has great significance for the diagnosis of thyroid problem. The study is also not according to the findings of Ladenson et al. have also considered that the TSH test as a criterion for the diagnosis of thyroid dysfunction, especially in cases of minimal thyroid failure (subclinical hypothyroidism) [1113]. The study has inconsistency with the findings of Evered et al. have been investigated that in case of mild hypothyroidism serum TSH also have been observed high and the values of T3 and T4 have remained in normal range. Such studies would be helpful to understand on the prevalence of diffuse goiter in different subjects and will also suggest the measures to minimize the goiter associated with its onset. It is further suggested that that the role of hormonal interactions in these patients may also be investigated with reference to their different metabolic disorders.

\section{Conclusion}

The present study concluded that prevalence of diffuse goiter may be dependent on the extent of hormonal dysfunctioning specifically normal and abnormal TSH levels. In future comprehensive investigation is warrant covering the epidemiological profile of the patients having diffuse goiter.

3 Schwartz CE, Stevenson RE (2007) The MCT8 thyroid hormone transporter and Allan-Herndon-Dudley syndrome. Best Pract Res Clin Endocrinol Metab 21: 307-321.

4 Surks MI, Hollowell JG (2007) Age-specific distribution of serumthyrotropin and antithyroid antibodies in the US population: implicationsfor the prevalence of subclinical hypothyroidism. J Clin Endocrinol Metab 92: 4575-4582. 
5 Oppenheimer JH, Koerner D, Schwartz HL, Surks MI (1972) Specific nuclear triiodothyronine binding sites in rat liver and kidney. J Clin Endocrinol Metab 35: 330-333.

6 Morreale de Escobar G, Obregon MJ, Escobar Del Rey F (2004) Role of thyroid hormone during early brain development. Eur J Endocrinol 151: 45-78.

7 http://emedicine.medscape.com/article/819692-overview

8 Suzuki S, Nisiho S, Takeda T, Komatsu M (2012) Gender-specific regulation of response to thyroid hormone in aging. Thyroid Res 5: 34-45.

9 Khan A, Khan MAM, Akhtar S (2002) Thyroid disorders, etiology and prevalence. Med Sci 2: 89-94.
10 Caldwell G, Gow SM, Sweating VM, Kellett HA, Beckett HJ, et al. (1985) A new strategy for thyroid function testing. Lancet 1 : 1117-1119.

11 Evren B (2012) Introduction to thyroid: anatomy and functions, thyroid and parathyroid diseases-new insights into some old and some new issues. Endocrinology 4: 45-78.

12 http://trove.nla.gov.au/work/28692053

13 Vander Pump MPJ (2005) The epidemiology of thyroid diseases. In: LE B and Utiger RD (ed.) Werner and Ingbar's the thyroid: a fundamental and clinical text ( $9^{\text {th }}$ edn.). Philadelphia: JB LippincottRaven pp: 398-496. 